\title{
Editorial: Sprachgeschichte als Kulturgeschichte - revisited
}

\author{
Constanze Spieß $\cdot$ Susanne Tienken
}

Online publiziert: 7. Juni 2019

(C) Springer-Verlag GmbH Deutschland, ein Teil von Springer Nature 2019

\section{Einleitung}

Als vor 20 Jahren der Sammelband »Sprachgeschichte als Kulturgeschichte « herausgegeben wurde (Gardt/Haß-Zumkehr/Roelcke 1999), konnte die deutschsprachige Sprachgeschichte zwar bereits sozialhistorische Bezugnahmen aufweisen (vgl. z. B. Reichmann 1985¹, von Polenz 1994, Hermanns 1995, Linke 1996), für eine Kulturalisierung der Sprachgeschichte wurde mit dem Band damals jedoch Programmatisches geleistet und befestigt, auch wenn dies heute nicht mehr im wissenschaftlichen Selbst-Bewusstsein der germanistischen Linguistik besonders prominent hervortritt. Letzteres dürfte erstens einer Art disziplinärem Versickerungsprozess geschuldet sein - der Grundgedanke, Sprachgeschichte als Kulturgeschichte (und nicht nur als Komplement) zu betreiben, ist mittlerweile weitgehend etabliert - und zweitens der Tatsache, dass sich das Spektrum sowie das Erkenntnisinteresse der Bezugswissenschaften seit 1999 rasant erweitert hat (dazu rezent Linke 2018; Czachur 2018; Schröter/Tienken/Ilg 2019).

Die im Titel der Einleitung angekündigte Revision von Sprachgeschichte als Kulturgeschichte im Sinne einer >nochmaligen Sichtung< oder >Prüfung auf Verwertbarkeit< gilt daher nur sekundär dem namensverwandten Sammelband, um einer fachin-

\footnotetext{
1 Der Aufsatz von Reichmann trug den Titel »Sprachgeschichte als Kulturgeschichte«. Der erwähnte Sammelband wurde zu seinen Ehren herausgegeben.

C. Spieß $(\bowtie)$

Institut für Germanistik, Karl-Franzens-Universität, Graz, Österreich

E-Mail: constanze.spiess@uni-graz.at

S. Tienken

Institut für slawische und baltische Sprachen, Finnisch, Niederländisch und Deutsch, Universität

Stockholm, Stockholm, Schweden

E-Mail: susanne.tienken@tyska.su.se
} 
ternen Vergesslichkeit entgegenzuwirken ${ }^{2}$, sie gilt vor allem der Herausbildung einer kulturanalytischen bzw. kulturbezogenen Sprachgeschichte, wie sie hauptsächlich in den vergangenen beiden Jahrzehnten stattgefunden hat. Dazu werden wir zunächst auf Kultur als sprachwissenschaftliches bzw. sprachhistorisches Untersuchungsfeld eingehen, um danach ausgewählte inner- und transdisziplinäre Bezüge darzulegen und abschließend einige Potentiale sowie Herausforderungen kulturgeschichtlichsprachgeschichtlicher Untersuchungen aufzuzeigen. Die Auseinandersetzung mit unterschiedlichen Aspekten einer linguistisch akzentuierten Kulturgeschichte setzt sich in den Einzelbeiträgen fort.

\section{Kultur als sprachgeschichtliches Untersuchungsfeld}

Der damalige Sammelband war dem wissenschaftlichen Unterfangen geschuldet, »den historiographischen Gegenstand über die Sozialgeschichte im engeren Sinne in die Kulturgeschichte zu erweitern« (Gardt/Haß-Zumkehr/Roelcke 1999, S. 1). Analog und fast zeitgleich $\mathrm{zu} »$ Sprachgeschichte als Kulturgeschichte« ist rückblickend die Forderung zu verzeichnen, Sprachwissenschaft insgesamt als Kulturwissenschaft zu betreiben oder zumindest zu verstehen (z. B. Auer 2000; später Gardt 2003; Linke 2003; Hornscheidt 2003, Wengeler 2006; Günthner/Linke 2006).

Dabei gerät ein Kulturbegriff in den Fokus, der sich des Normativen, Aufwertenden, >Hochkulturellen $<$ entledigt und stattdessen instrumentell umgedeutet und genutzt werden kann und zwar in einer Weise, in der »Kultur zu einem analytischen Begriff umgewandelt [wird], so dass die kulturellen Normen selbst und ihre Handhabung durch Menschen zum Gegenstand einer Wissenschaft werden können« (Haß-Zumkehr 1999, S. 250). Bei einer solchen analytischen Umdeutung ist ein verändertes, disziplinär zu verankerndes Bewusstsein von zentraler Bedeutung, bei der ein »System sowohl sprachlicher als auch nicht-sprachlicher Zeichen « als Untersuchungsfeld in den Blick genommen wird, anhand dessen sich »die Angehörigen einer konkreten Gesellschaft oder gesellschaftlichen Gruppe [...] die Welt und ihre Situation in ihr deuten und ihr Handeln orientieren « (Gardt/Haß-Zumkehr/Roelcke 1999, S. 2). Selbst wenn Betrachtungen zum Verhältnis von Sprache und Kultur schon bei Herder oder im sprachlichen Idealismus von Humboldt erscheinen, sowie auch im 19. Jahrhundert etwa von Hermann Paul mit dem Postulat von Sprachwissenschaft als Kulturwissenschaft weitergeführt werden (vgl. Tienken 2015, S. 465; Cherubim 2016, S. 692; Luginbühl im Druck), haben wir somit mit dem Sammelband von Gardt/Haß-Zumkehr/Roelcke eine der ersten forschungsgemeinschaftlichen Publikationen vor uns, in der eine »theoretische Wahrnehmung der Kulturalitätsdimension der Sprache« zu verzeichnen ist (Jäger et al. 2016, S. 1). Die Autor*innen des Sammelbands von 1999 dürften also dazu beigetragen haben, dass in der germanistischen Linguistik heute weitgehend Einigkeit darüber besteht, dass Sprache und Kultur zwei Größen sind, die nicht als einander beigeordnet, sondern als interdependent, ja in ihrer gegenseitigen Emergenz zu untersuchen sind (vgl. Günthner/Linke 2006; Tienken 2008; Schröter/Tienken/Ilg 2019).

\footnotetext{
2 Wir danken Jörg Kilian für dieses Stichwort.
} 
Sprachhistorisch beziehungsweise linguistisch-kulturanalytisch Forschende sind aber dennoch lange mit der Frage weitgehend allein gelassen worden, was denn eigentlich zu untersuchen ist, wenn es um Kultur geht. Fritz Hermanns (1999, S. 379) nennt gar noch die wenig hoffnungsfrohe Zahl von 141 Definitionen von Kultur. Zur übergreifenden Orientierung lassen sich jedoch drei Ansätze ausmachen - ein kognitiver, ein handlungsorientiert-semiotischer sowie ein konstruktivistischer Ansatz (vgl. Auer 2000; Tienken 2008, S. 49-53). Die Diversität unterschiedlicher Zugänge hat das kulturanalytisch-linguistische Feld zu eben einer solchen analytischen Instrumentalisierung geführt, wie von Haß-Zumkehr (1999) eingefordert. Die zentrale Frage lautet folglich nicht mehr, wie Kultur zu definieren ist, sondern eher, wie Kulturkonzepte genutzt werden können, um vergangene Lebens-und Sprechweisen zu verstehen. Ein Überblick über die Publikationen linguistischer Kulturanalyse der vergangenen Jahrzehnte zeigt, dass insbesondere ein semiotisch-handlungsorientiertes Kulturkonzept nutzbar gemacht worden ist (vgl. Schröter/Tienken/Ilg 2019). Ausgegangen wird dabei in der Regel von Clifford Geertz' anthropologischem »webs of significance« (Geertz 1973, S. 5), bei dem Kultur als ein von Menschen hergestelltes Netz von Sinnzuweisungen zu denken ist. In der linguistischen Kulturanalyse wird zudem vorausgesetzt, dass dieses Netz im sozialen, sprachlichen Miteinander von Menschen gemacht wird. Kommunikation ist folglich der Ort der Emergenz, des Aushandelns und des Weiterführens von Kultur. Die Zeichenhaftigkeit und somit auch die Lesbarkeit einer >Kultur als Text< im Sinne von Bachmann-Medick (1996, S. 10) offenbart sich für sprachwissenschaftlich Forschende entsprechend in der sprachlichen Form und Performanz (vgl. Tienken 2015, S. 467; Linke 2016, S. 356; Holly/Jäger 2016, S. 946). Gerade im historischen Kontext ist Kultur in einem solchen Verständnis nicht das Bekannte oder Erklärende, sondern eher das noch zu Erschließende. Aus diesem Grund ist es neben der tiefgehenden Befragung historischer Nachbardisziplinen oftmals notwendig, >das Kulturelle < überhaupt erst einmal analytisch sichtbar zu machen. Dabei kann die sprachgeschichtliche Forschung die Erkenntnis nutzen, dass sprachliche Muster intersubjektiv wahrgenommene Relevanzbereiche anzeigen. Dies gilt für Muster sämtlicher linguistischer Analyseebenen (vgl. Tienken 2015; Holly/Jäger 2016; Luginbühl i. Dr.). Der wiederholte, musterhafte Gebrauch von bestimmten Formen ist ein Effekt und die Voraussetzung von Vergesellschaftung zugleich. Zeichen können überhaupt erst sinnhaft und somit $>$ kulturell werden, wenn sie repetitiv in Gebrauch genommen werden und ein geteilter Erwartungshorizont bezüglich dessen besteht, wie und wofür die Zeichen eingesetzt werden. Dies gilt für die heutige Zeit und ist auch für historische Lebenswelten nicht anders. Für eine kulturanalytische historische Sprachwissenschaft ist dabei relevant, dass sprachliche Muster sowohl symbolische (qua Übereinkunft) als auch indexikalische (qua Gebrauchsspuren) Bedeutungen tragen können. Um diese rekonstruieren zu können, sind - soweit möglich - die Beteiligtenrollen, Medialität und Materialität sowie die Verortung im (sozialen) Raum zu berücksichtigen. Der aus der Gesprächsanalyse entlehnten »Gretchenfrage « Why that now? (Linke 2018, S. 263) müssten also folglich auch noch die beiden Fragen Why them? und Why there? hinzugefügt werden. Dass dabei alle verfügbaren Quellen unter den methodischen Leitprinzipien der Kontrastierung und Triangulierung hinzugezogen werden müssen, liegt nahe (vgl. Tienken 2015). 


\section{Disziplinäre Bezüge, Potentiale und Herausforderungen einer kulturanalytisch orientierten Sprachgeschichte}

Die Herausbildung einer kulturanalytisch orientierten Sprachgeschichte ist einerseits im Kontext eines größeren wissenschaftlichen Paradigmenwechsels im Zuge des >Linguistic Turn $<$ und des >Cultural Turn $<$ zu verstehen, bei denen die Wechselbeziehung der beiden Größen Sprache und Kultur in den Fokus gestellt wird. Das Interesse, das vor dem Hintergrund dieser >Turns < gerade die Geschichtswissenschaften der Ausdeutung von sprachlichen Formen entgegen gebracht haben und weiterhin auch tun $^{3}$, ließe eine wesentlich engere Zusammenarbeit zu, als es momentan der Fall ist. Die Herausbildung von Kulturwissenschaft als Fach hat zudem die Beschäftigung mit Performanz und Performativität, mit Körperlichkeit, Medialität und Materialität, mit Raum-Zeitlichem und Praktiken sowohl in den Geschichtswissenschaften als auch in der kulturanalytischen Linguistik befördert (vgl. Schröter/ Tienken/Ilg 2019). Das z. B. von Angelika Linke (2003, S. 56) eingeforderte interdisziplinäre Projekt ist allerdings immer noch nicht vollständig eingelöst.

Die kulturanalytisch orientierte Sprachgeschichte ist innerhalb der Sprachwissenschaft mit solchen Teildisziplinen eng verflochten, die teilweise auch ohne kulturanalytischen Anspruch musterhafte Formen sowie Bedeutungszuweisungen analysieren. Dazu gehören vor allem historische Lexikologie und Semantik, Phraseologie und Konstruktionsgrammatik, Pragmatik, Textlinguistik, Gesprächsforschung, sowie auch Medienlinguistik und Stilistik. Auch politolinguistische und diskursanalytische Forschung ist mittlerweile eng mit einem genuin kulturanalytisch-(zeit)sprachgeschichtlichen Erkenntnisinteresse verbunden (vgl. Schröter/ Tienken/Ilg 2019; Luginbühl i. Dr.; Spieß 2011, 2018)4.

Mit dem gemeinsamen Interesse an der Soziokulturalität von Sprache ist die historische Soziolinguistik zweifelsohne eine der wichtigsten Schwesterdisziplinen der kulturanalytisch orientierten Sprachgeschichte, die anders als die kulturanalytische Linguistik auch außerhalb der germanistischen Linguistik weitgehend etabliert ist. Der Unterschied zwischen historischer Soziolinguistik und historisch ausgerichteter kulturanalytischer Linguistik bestand lange Zeit darin, dass sich die Soziolinguistik für Effekte gesellschaftlicher Ordnung und Phänomene auf Sprache und sprachlichen Wandel interessiert, die kulturanalytische Linguistik hingegen eher dafür, inwiefern Sprache Welt hervorbringt und Sinngebungen anzeigt. Es wird mit Spannung zu verfolgen sein, inwiefern indexikalische Ansätze der sogenannten 3. Welle der Soziolinguistik dazu beitragen werden, diesen Unterschied einzuebnen (vgl. Russi 2016).

Trotz dieser Verbindungen weist die kulturanalytisch orientierte Sprachgeschichte spezifische Potentiale auf: Eine kulturanalytisch orientierte Sprachgeschichte ist, im weiter gefassten Sinne, Forschung am Menschen. Sie ermöglicht es und hat zum Ziel, Sprachgebrauchssituationen in ihrer kulturellen Bedeutung zu rekonstruieren, den gemeinschaftlich erzeugten Sinn vergangener Äußerungen nachzuzeichnen und

\footnotetext{
3 International besonders einflussreich, wenn auch nicht unumstritten, war das gigantische Projekt von Brunner/Conze/Koselleck (1972-1992) zu den geschichtlichen Grundbegriffen.

4 Einen ausführlichen Überblick bietet Linke 2018.
} 
sprachliche Individualerscheinungen vor der Folie von Mustern deuten zu können. Eine kulturanalytisch orientierte Sprachgeschichte ergründet das Sagbare einer Epoche und damit letztlich auch das Machbare (vgl. Steinmetz 1993), die Begrenzungen und Möglichkeiten von Menschen in der Vergangenheit. Es geht um die »Gestaltung zur Welt « in Cassirers (2010, S. 9; Hervorh. i.O.) Sinne. Dies bringt eine Erfahrung von »Touching the Past « (van der Wal/Rutten 2013, S. 1) mit sich, die empirisch das Zeug hat, heutige Lebensentwürfe, Gefühle, Sichtweisen sowie Inszenierungen von Macht und Ohnmacht zu früheren in Beziehung zu setzen oder ihnen gegenüber zu stellen. Und die theoriebildend dazu angetan ist, die sprachliche Verfasstheit von Welt (und sich selbst) als Grundbedingung menschlichen Daseins, als universales Prinzip zu verstehen. Die den Menschen als Akteur (und nicht nur Kultur) in die Linguistik zurückholt. Dies ist zum einen reizvoll für die Forschungsgemeinschaft, zum anderen aber auch in äußerstem Maße gesellschaftsrelevant. Dass der gut $20 \mathrm{Jah}-$ re vor Brexit erschienene Sammelband »Sprachgeschichte als Kulturgeschichte« mit der Bemerkung von Christopher Wells endet, dass »es in England Kreise gibt [...], die der politischen Vereinigung Europas mit extremer Skepsis entgegensehen« (Wells 1999, S. 418), ist vor diesem Hintergrund besonders bemerkenswert.

Die Herausforderungen, die eine kulturanalytische Sprachgeschichte zu bemeistern hat, sind zunächst einmal gesellschaftlicher Art. Genau wie die Linguistik insgesamt muss sie sich die Frage stellen, auf welche Weise sie in Zeiten zunehmender Mediatisierung ihre Erkenntnisse verbreiten kann und soll und wie sie kommenden Generationen einen verantwortungsbewussten Umgang mit historischen Fakten vermitteln kann. Da sie mitunter tatsächlich auch Äußerungen existierender, womöglich sogar noch lebender Menschen analysiert, wird zudem eine Weiterentwicklung forschungsethischer Maßstäbe unerlässlich sein.

Weiterhin stellen sich methodische Herausforderungen - Wie verhalten sich qualitativ-hermeneutische Verfahren und quantitative Verfahren zueinander? Datenbanken (falls überhaupt vorhanden) und digitale Suchverfahren können für die Entdeckung von frequenten Mustern zwar erfolgreich genutzt werden, aber wie lassen sich deren Kontextualisierungspotentiale und ihre Sozialität, ihre Signifikanz in einer historischen Verortung rekonstruieren? Ferner ist zu überlegen, was bei mangelnder Serialität von Quellen zu tun ist. Triangulierende oder auch rhizomatisch angelegte Verfahren, die der Diskursanalyse und der neueren Soziolinguistik entlehnt sind (vgl. Bohlin 2018), müssten entsprechend implementiert und weiterentwickelt werden. Sicher ist, dass eher eine Art methodische Werkzeugkiste zu entwickeln sein wird als ein festes Analysemodell, wenn die kulturanalytisch orientierte Sprachgeschichte ihren offenen Erkenntnishorizont behalten will.

Da mit der Interpretation von sprachlichen Mustern gearbeitet wird, wird eine wesentliche Herausforderung weiterhin darin bestehen, sich auf die soziokulturellen Bedingungen, das Setting von historischen sprachlichen Äußerungen fachkundig einzulassen. Ohne eine interdisziplinäre Vernetzung mit den historischen Nachbarwissenschaften sowie auch der historischen Philologie laufen nämlich Deutungen Gefahr, in anachronistischer Weise eher die heutigen Erfahrungswelten und Bewertungen sozialer Handlungen zu spiegeln als Zugang zu historischen Sinngebungsverfahren zu bieten (vgl. Bergs 2013). Dies ist bereits von Klaus Mattheier (1999, S. 16-18) stark kritisiert worden. Das Betreiben einer >Sprachgeschichte von unten< 
(Elspaß 2005) mag diese Kritik zwar mildern, das grundlegende Probleme einer historisch adäquaten Kontextualisierung bleibt aber bestehen.

\section{$4 \mathrm{Zu}$ den Beiträgen}

Das Themenheft entspringt einem gleichnamigen Symposium des schwedischen Netzwerkes für Sprach- und Kulturforschung, das im Juni 2017 an der Universität Stockholm stattfand. Die onomastischen Beiträge von Damaris Nübling und Friederike Kreil sowie ein wissenschaftshistorischer Beitrag von Michael Prinz erscheinen auf Wunsch der Verfasser*innen in anderen Publikationen, gleichwohl haben sie sich auf dem Symposium aktiv zu der Sichtung des Status Quo kulturwissenschaftlich orientierter Sprachgeschichte beigetragen. Ihnen sei an dieser Stelle herzlich gedankt.

Die Beiträge beziehen ihre weit unterschiedliche Empirie aus mehreren Jahrhunderten, vom Mittelalter bis zur heutigen Zeit. Allen gemeinsam ist jedoch, dass sie sich mit der jeweiligen sprachlichen Verfasstheit von Welt auseinandersetzen und einigen Schlüsselfragen der kulturanalytischen Linguistik historisch nachgehen: der Ausdeutung symbolischer Formen und Musterbildungen, den gesellschaftlichen Dimensionen von Sprachgebrauch, der kulturellen Konzeptualisierung und Instrumentalisierung von Gefühlen, der Ideologisierung von Sprache sowie der sprachlichen Hervorbringung von Sozialgefügen.

In seinem Beitrag »Morphosemantik und Kulturanalyse. Substantivkomposita und kulturelles Sinngebungspotential in frühmittelalterlichen Schultexten Notkers III.« geht Nicolaus Janos Raag der Frage nach, in welcher Weise Wortbildungen an Sinngebungsprozessen mitwirken. Gegenstand seiner Analyse sind die frühmittelalterlichen wissensvermittelnden Texte Notkers III. Dabei handelt es sich um didaktische Bearbeitungen und Übersetzungen aus dem Lateinischen. Das frühmittelalterliche Deutsch ist insofern besonders geeignet, um solche Sinngebungsprozesse zu rekonstruieren, da es sich zu dieser Zeit gerade von einer mündlichen Alltagssprache hin zu einer Schriftsprache entwickelt. Die damit verbundene Ausdifferenzierung der deutschen Sprache zeigt sich insbesondere am Wortschatz, u.a. in der Benennung von Sachverhalten und Konzepten, die im Alltagssprachgebrauch bislang keine Verwendung fanden, aber auch in der Typisierungsleistung durch Komposita, insofern aus den Übersetzungen resultierende Konzepte durch Substantivkomposita in die bekannte Welt eingeordnet werden. Augenfällig wird dies bei Notkers didaktischen Übersetzungen, die - so der Autor - das Ziel verfolgten, den lateinischen Text nicht einfach nur zu übersetzen, sondern verständlich zu machen. Raag zeigt an seinem Korpus, dass es zwei Muster sind, die mithilfe von Substantivkomposita an der kulturellen Sinnerzeugung beteiligt sind: zum einen die erklärende Übersetzung und zum anderen die Zusammenfassung komplexer Inhalte. Damit tragen Substantivkomposita einen wesentlichen Anteil an der kommunikativen Gestaltung von Kultur.

Dem kulturellen Ordnungsmedium der Liste widmet sich Michelle Waldispühl mit ihrem Beitrag »Die Liste als Ordnungsmedium in mittelalterlichen Libri vitae«. Die Autorin konstatiert, dass Listen in allen Epochen und Schriftkulturen 
$\mathrm{zu}$ verschiedenen kommunikativen Zwecken eingesetzt wurden; ihre funktionale Gemeinsamkeit liegt allgemein gesprochen in einem gewissen Ordnungspotenzial. Waldispühl fasst Listen als Textsorten und unterzieht diese einer mehrdimensionalen Analyse, die das Zusammenspiel von Formgebundenheit, Thematizität, Funktionalität sowie situativ-kontextueller Verortung (in gesellschaftliche, institutionelle, politische und soziale Zusammenhänge) in den Blick nimmt. Dabei stellt sie eine Vielfalt an formalen und inhaltlichen Prinzipien sowie kommunikativen Zwecken fest, die ihre je spezifische Begründung jeweils aus diesem Zusammenspiel von sprachlicher Gestaltung, inhaltlicher Ausrichtung, kommunikativem Zweck und institutionellem wie politisch-gesellschaftlichem Kontext erfahren. Es zeigt sich auch hier sehr deutlich, dass sich kulturelle und soziale Praktiken sprachlich manifestieren. Ändert sich in diesem Zusammenspiel beispielsweise ein kontextuell-situativer Faktor, so hat diese Veränderung Auswirkungen auf alle anderen Beschreibungsdimensionen der Textsorte Liste. Listen in den Libri vitae können, so Waldispühl, als »Kulturpraxis und Medium sozialer Ordnung « aufgefasst werden, da durch sie soziale Beziehungen und soziale Ordnungen nicht nur dargestellt, sondern in erster Linie erzeugt werden.

Dessislava Stoeva-Holm befasst sich in ihrem Beitrag »Huldigung des Gefühls im 17. Jahrhundert.« mit dem besonderen Verhältnis von Sprache und Musik am Beispiel der multikodalen Textsorte der Gelegenheitskomposition, wobei sie den Fokus auf die Sprache legt. Stoeva-Holm zeigt, wie sehr solche Kompositionen im Hinblick auf gesellschaftspolitische Zwecke genutzt wurden und politisch-soziale sowie kulturelle Funktionen übernommen haben; u.a. wurden solche Gelegenheitskompositionen erzeugt, um dem König zu huldigen, seine Herrschaft zu legitimieren, ihn zu loben, seine Macht zu inszenieren, um zu unterhalten und darüber hinaus aber auch die Erwartungen an ihn zu formulieren und ihn zugleich seiner Verpflichtungen als Herrscher zu gemahnen, was häufig aber indirekt geschah. Dass in diesen Zusammenhängen Emotionen, die sich auf verschiedene Weise sprachlich manifestieren können, eine wichtige Rolle einnehmen, zeigt Stoeva-Holm eindrücklich am Beispiel von Hochzeitskompositionen aus der Dübensammlung, einem Archiv für barocke Vokal- und Instrumentalmusik. Die sprachlichen Manifestationen von Emotionen geben dabei Aufschluss darüber, wie Gefühle im Zeitalter des Barock konzeptualisiert wurden. Das Zusammenspiel von Sprache und Musik verweist der Autorin zufolge darüber hinaus auf verschiedene Funktionen von Gelegenheitskompositionen im Hinblick auf Affektgestaltung und -vermittlung, wobei es unterschiedliche Relationen zwischen Musik und sprachlicher Repräsentation gibt.

Einen praxeologischen Ansatz vertritt Angelika Linke in ihrem Beitrag »Schreiben als Beziehungspraxis. Frauenbriefe aus dem Zürcher Bürgertum 1760-1800. Ein Entwurf «. Sie untersucht den Zusammenhang von privater Schriftlichkeit und Beziehungsperformanz anhand eines Belegkorpus von Briefen junger bürgerlicher Frauen aus der zweiten Hälfte des 18. Jahrhunderts in Zürich. Dabei gerät auch die Materialität der verwendeten Empirie in den Fokus: Briefe sind als »Materialisierungen interaktiven sprachlichen Handelns im Verdauerungsmedium der Schrift« zu charakterisieren, anhand derer historische Beziehungsformen nachgezeichnet werden können. Da Briefe zudem zwar epochal und soziokulturell variable, aber dennoch weitgehend überindividuelle, gattungsgeprägte Formen sind, kann eine Rekonstruk- 
tion auch anhand kleinerer Korpora vollzogen werden. Die beständige (Selbst-)Appellation als Freundin sowie die Thematisierung von Freundschaft sowie deren stilistische Überhöhung lässt das soziokulturelle Konzept bürgerlich-weiblicher Jugendfreundschaft hervortreten. Nicht zuletzt kann auch der Gattung Brief eine beziehungskonstitutive Funktion zugewiesen werden. Die Plausibilität der Ergebnisse wird zusätzlich durch eine Materialtriangulierung gesichert, da neben dem Zürcher Briefkorpus auch zeitgenössische Brieflehren untersucht werden. Die Analyse von Anrede und Selbstbezeichnungen sowie von Beziehungsbezeichnungen und sprachlichem Duktus insgesamt können auf diese Weise mit einem normativen Diskurs in Bezug gesetzt werden.

In ihrem Beitrag »Phraseologismen als Zeit- und Kulturdokumente - der Büchmann im Nationalsozialismus « befasst sich Carolin Krüger mit einem Vergleich zweier Ausgaben (1925 und 1937) der Zitatensammlung Georg Büchmanns. Grundannahme ihrer Analyse ist die Auffassung, dass Phraseologismen und geflügelte Worte verdichtete Kulturzeichen sind, in denen sich Weltanschauungen bzw. Ideologien sprachlich manifestieren und denen Erfahrungen, Überzeugungen oder Stereotype zugrunde liegen können. Der Büchmann galt im 19. Jahrhundert insbesondere in Kreisen, die sich als bildungsaffin konstruierten, als viel zitiertes Nachschlagewerk, um Bildungsaffinität zu demonstrieren. Auch die Nationalisozialisten verwendeten den Büchmann, vorwiegend aber, um die Zitate und geflügelten Worte für ihre Propagandazwecke zu gebrauchen. Der Vergleich der beiden Ausgaben macht deutlich, dass Wörterbücher kulturelle Prägungen erfahren; Wörterbücher im allgemeinen und das Zitatwörterbuch im Besonderen vermitteln und konstruieren kulturelle Orientierung. Durch den Vergleich der beiden Ausgaben zeigt Krüger eindrücklich, dass der Büchmann durch die Nationalsozialisten eine ideologische Umfärbung erfahren hat, entweder durch Weglassung, durch Hinzufügung oder durch Umformulierungen von Lemmata.

Unterschiedliche Benennungen eines Sachverhaltes weisen auf weltanschauliche Perspektiven, die dem Sachverhalt/Gegenstand/der Idee zugrunde liegen. Sie sind zugleich Ausweis kultureller Perspektiven und der sprachlichen Konstruktion von Wirklichkeiten. Ausgehend von einem korpuslinguistisch informierten diskurslinguistischen Ansatz geht Charlotta Seiler Brylla in ihrem Beitrag »Strategische Benennungen. Zur Sprachgeschichte des geteilten Deutschland in einem deutschschwedischen Kontext « auf schwedische und deutsche Bezeichnungen für die beiden Deutschen Staaten 1949-1989 im öffentlichen Diskurs ein und analysiert die mit den zeittypischen Bezeichnungspraktiken verbundenen Handlungszwecke und diskursiven Strategien. Diese geben Aufschluss über die zugrundeliegenden Ideologien, die Beziehungen der Staaten untereinander und schließlich auch über die in den sprachlichen Praktiken manifeste kulturelle Perspektivität, die in den unterschiedlichen staatlichen Systemen begründet liegt. Die Dimension der zeitgeschichtlichen Analyse des Sprachmaterials zeigt zudem, wie sich Benennungspraktiken im Laufe der Zeit zwischen 1949 und 1989 wandelten, so dass durch die verschiedenen Bezeichnungen zum Ausdruck gebracht wird, wie einerseits die Beziehung Schwedens zu den beiden deutschen Staaten konstruiert wurde bzw. wie sich Schweden zu den jeweiligen deutschen Staaten politisch positionierte; andererseits zeigt sich daran 
aber auch, wie die beiden deutschen Staaten ihre jeweilige Beziehung zu Schweden konzeptualisierten und um die Beziehung zu Schweden kämpften.

Gesellschaftliche Ordnungsmuster der Geschlechtsidentitäten, der Geschlechterkonstruktionen und des Verhältnisses der Geschlechter untereinander unterliegen einem kulturellen Wandel, wenngleich dieser Wandel sich nicht immer deutlich oder explizit zeigt bzw. zeigen muss. Änderungen in der Auffassung von Geschlecht und der Geschlechterkonstruktionen schlagen sich dementsprechend mehr oder weniger deutlich auf verschiedenen sprachstrukturellen Ebenen nieder. Hanna Acke widmet sich in ihrem Beitrag »Sprachwandel durch feministische Sprachkritik: Geschlechtergerechter Sprachgebrauch an den Berliner Universitäten « der Frage, inwiefern sich geschlechtergerechter Sprachgebrauch (als ein Ausweis der Geschlechterkonstruktion und als eine Ausprägung der Auffassung vom Verhältnis der Geschlechter) im Kommunikationsbereich Universität in einem Prozess der Normierung befindet. Den Normierungsprozess untersucht sie auf der Ebene der Verwendung sprachlicher Formen für Personenbezeichnungen. Dabei geht sie auf die verschiedenen grammatikalischen Möglichkeiten geschlechtergerechter Bezeichnungspraktiken ein. Gegenstand ihrer Untersuchung sind Richtlinien, Leitfäden und Styleguides der drei Berliner Volluniversitäten, in denen Aussagen zum geschlechtergerechten Sprachgebrauch gemacht werden.

Die Beiträge zeigen in der Zusammenschau, was Sprach(gebrauchs)geschichte, die als Kulturgeschichte betrieben wird, leisten kann und unter welchen Prämissen sie heute arbeitet. Wir haben es mit qualitativ angelegten Analysen zu tun, bei denen ein (vorrangig) semiotischer Kulturbegriff weiterhin genutzt werden kann, um relevante Phänomene an der sprachlichen Oberfläche aufspüren und deuten zu können. Sie zeigen aber auch, dass sich das Inventar und somit die Deutungsmöglichkeiten der linguistischen Kulturanalyse in den letzten 20 Jahren erheblich erweitert hat und weiterhin erweiterbar bleibt. Dazu gehören die analytische Wahrnehmung und Ausdeutung von Materialität und der Multimodalität der vorgefundenen historischen Quellen, die Nutzung linguistischer Ansätze wie kritischer Diskursanalyse oder von Membership Categorization Analysis, die Kontrastierung unterschiedlicher Muster, sowie das Ausschöpfen ursprünglich soziologischer Konzepte wie dem der Praktiken oder der kommunikativen Gattung, der Institutionalisierung oder der Normalisierung.

Die Relevanz von theoretischer Revision und auch von Neusichtung und Neuinterpretation historischen Materials, sowie die Bedeutsamkeit eines kritischen Blicks auf die eigene, auch noch junge, Wissenschaftsgeschichte werden somit in einem kleinen Rahmen eingängig vorgeführt. Das Themenheft erhellt ferner, auf welche Weise kulturanalytische Sprachgeschichtsforschung eine fruchtbare Synthese unterschiedlicher disziplinärer Zugangsweisen und Fragestellungen leisten kann und ermuntert zu einer verstärkt transdisziplinären Weiterarbeit am Feld.

\section{Literatur}

Auer, Peter: »Die Linguistik auf dem Weg zur Kulturwissenschaft«. In: Freiburger Universitätsblätter 147 (2000), S. 55-68. 
Bachmann-Medick, Doris: »Einleitung«. In: Dies. (Hg.): Kultur als Text. Die anthropologische Wende in der Literaturwissenschaft. Frankfurt a.M. 1996, S. 7-64.

Bergs, Alexander: »The Uniformitarian Principle and the Risk of Anachronisms in Language and Social History«. In: Juan Manuel Hernández-Campoy/Juan Camilo Condo-Silvestre (Hg.): The Handbook of Historical Sociolinguistics. Blackwell 2013, S. 80-98.

Bohlin, Henrike: »Jugendsprache im norddeutschen Raum - ein Werkstattbericht«. In: Eva Neuland/ Benjamin Könning/Elisa Wessels (Hg.): Jugendliche im Gespräch: Forschungskonzepte, Methoden und Anwendungsfelder aus der Werkstatt der empirischen Sprachforschung. Berlin 2018, S. 255-274.

Brunner, Otto/Conze, Werner/Koselleck, Reinhart: Geschichtliche Grundbegriffe. 6 Bände. Stuttgart 1972-1992.

Cassirer, Ernst: Philosophie der symbolischen Formen. Erster Teil. Die Sprache. Hamburg 2010.

Cherubim, Dieter: »Kulturwissenschaftliche Orientierung in der Sprachwandeltheorie«. In: Ludwig Jäger/Werner Holly/Peter Krapp/Samuel Weber/Simone Heekeren (Hg.): Sprache - Kultur - Kommunikation. Ein internationales Handbuch zu Linguistik als Kulturwissenschaft. Berlin/Boston 2016, S. 688-701.

Czachur, Waldemar: »Kulturwissenschaftlicher Denkstil in der germanistischen Linguistik. Motivationen, Beharrungstendenzen und Entwicklungsrichtungen«. In: Christiane Andersen/Ulla Fix/Jürgen Schiewe (Hg.): Denkstile in der deutschen Sprachwissenschaft. Bausteine einer Fachgeschichte aus dem Blickwinkel der Wissenschaftstheorie Ludwik Flecks. Berlin 2018, S. 137-166.

Elspaß, Stephan: Sprachgeschichte von unten. Untersuchungen zum geschriebenen Alltagsdeutsch im 19. Jahrhundert. Tübingen 2005.

Gardt, Andreas/Haß-Zumkehr, Ulrike/Roelcke, Thorsten: »Vorwort«. In: Dies. (Hg): Sprachgeschichte als Kulturgeschichte. Berlin/New York 1999, S. 1-10.

Gardt, Andreas: »Sprachwissenschaft als Kulturwissenschaft«. In Ulrike Haß/Christoph König (Hg.): $L i$ teraturwissenschaft und Linguistik von 1960 bis heute. Göttingen 2003, S. 271-288.

Geertz, Clifford: »Thick description: Toward an interpretative theory of culture«. In: Ders. (Hg.): The interpretation of cultures: Selected essays. New York 1973, S. 3-30.

Günthner, Susanne/Linke, Angelika: »Einleitung: Linguistik und Kulturanalyse. Ansichten eines symbiotischen Verhältnisses«. In: Zeitschrift für Germanistische Linguistik 34/2 (2006), S. 1-27.

Haß-Zumkehr, Ulrike: »Die kulturelle Dimension der Lexikographie. Am Beispiel der Wörterbücher von Adelung und Campe«. In: Andreas Gardt/ Ulrike Haß-Zumkehr/Thorsten Roelcke (Hg): Sprachgeschichte als Kulturgeschichte. Berlin/New York 1999, S. 247-265.

Hermanns, Fritz: »Sprachgeschichte als Mentalitätsgeschichte. Überlegungen zu Sinn und Form und Gegenstand historischer Semantik«. In: Andreas Gardt/Klaus J. Mattheier/Oskar Reichmann (Hg.): Sprachgeschichte des Neuhochdeutschen. Gegenstände, Methoden, Theorien. Tübingen 1995, S. 69-101.

Hermanns, Fritz: »Sprache, Kultur und Identität. Reflexionen über drei Totalitätsbegriffe«. In: Andreas Gardt/ Ulrike Haß-Zumkehr/Thorsten Roelcke (Hg): Sprachgeschichte als Kulturgeschichte. Berlin/New York 1999, S. 351-391.

Holly, Werner/Jäger, Ludwig: »Aspekte einer kulturwissenschaftlichen Linguistik«. In: Ludwig Jäger/ Werner Holly/Peter Krapp/Samuel Weber/Simone Heekeren (Hg.): Sprache - Kultur - Kommunikation. Ein internationales Handbuch zu Linguistik als Kulturwissenschaft. Berlin/Boston 2016, S. 944-956.

Hornscheidt, Antje: »Sprach(wissenschaft)liche Kulturen. Plädoyer für eine linguistische Partizipation an einem konstruktivistisch begründeten, kulturwissenschaftlichen Projekt transdisziplinärer Forschung am Beispiel der Interkulturellen Kommunikation«. In: Linguistik online 14/2 (2003), S. 1-27.

Jäger, Ludwig/Holly, Werner/Krapp, Peter/ Weber, Samuel/Heekeren, Simone: »Einleitung«. In: Dies. (Hg.): Sprache - Kultur - Kommunikation. Ein internationales Handbuch zu Linguistik als Kulturwissenschaft. Berlin/Boston 2016, S. 1-5.

Linke, Angelika: Sprachkultur und Bürgertum. Zur Mentalitätsgeschichte des 19. Jahrhunderts. Stuttgart/ Weimar 1996.

Linke, Angelika: »Sprachgeschichte - Gesellschaftsgeschichte - Kulturanalyse«. In: Helmut Henne/Horst Sitta/Herbert Ernst Wiegand (Hg.): Germanistische Linguistik: Konturen eines Fachs. Tübingen 2003, S. 26-65.

Linke, Angelika: »Einführung: Kommunikation und Kulturalität«. In: Ludwig Jäger/Werner Holly/Peter Krapp/Samuel Weber/Simone Heekeren (Hg.): Sprache - Kultur - Kommunikation. Ein internationales Handbuch zu Linguistik als Kulturwissenschaft. Berlin/Boston 2016, S. 351-368.

Linke, Angelika: »Kulturhistorische Linguistik«. In: Arnulf Deppermann/Silke Reineke (Hg.): Sprache im kommunikativen, interaktiven und kulturellen Kontext. Berlin/Boston 2018, S. 347-383. 
Luginbühl, Martin: »Sprache und Kultur in der Kontrastiven Medienlinguistik: Vom Ländervergleich zur Analyse kulturell verdichteter Praktiken«. In: Hans Giessen/Hartmut Lenk/Susanne Tienken/Liisa Tiittula (Hg.): Medienkulturen - Multimodalität und Intermedialität. Bern (i.Dr.).

Mattheier, Klaus: »Sprachhistoriker als Soziologen. Über sprachwissenschaftliche Versuche zur Strukturierung sozialer Gemeinschaften «. In: Andreas Gardt/Ulrike Haß-Zumkehr/Thorsten Roelcke (Hg): Sprachgeschichte als Kulturgeschichte. Berlin/New York 1999, S. 11-18.

Polenz, Peter von: Deutsche Sprachgeschichte vom Spätmittelalter bis zur Gegenwart. Band II: 17. und 18. Jahrhundert. Berlin/New York 1994.

Reichmann, Oskar: »Sprachgeschichte als Kulturgeschichte. Historische Wortschatzforschung unter gegenwartsbezogenem Aspekt«. In: Alois Wierlacher (Hg.): Das Fremde und das Eigene. Prolegomena zu einer interkulturellen Germanistik. München 1985, S. 111-122.

Russi, Cinzia: »Introduction«. In: Dies. (Hg.): Current Trends in Historical Sociolinguistics. Warsaw 2016, S. $1-18$.

Schröter, Juliane/Tienken, Susanne/Ilg, Yvonne: »Linguistische Kulturanalyse. Eine Einführung «. In: Juliane Schröter/Susanne Tienken/Yvonne Ilg/Noah Bubenhofer/Joachim Scharloth (Hg.): Linguistische Kulturanalyse. Berlin/Boston 2019, S. 1-27.

Spieß, Constanze: Diskurshandlungen. Theorie und Methode linguistischer Diskursanalyse am Beispiel der Bioethikdebatte. Berlin/Boston 2011.

Spieß, Constanze: » Deutschland muss Deutschland bleiben< - Sprachliche Selbst- und Fremdpositionierungsaktivitäten im Kontext politischer Äußerungen über Migration am Beispiel des Ausdrucks Leitkultur.« In: Kulturwissenschaftliche Zeitschrift 3/1 (2018), S. 25-55. https://doi.org/10.2478/kwg2018-0003

Steinmetz, Willibald: Das Sagbare und das Machbare. Zum Wandel politischer Handlungsspielräume England 1789-1867. Stuttgart 1993.

Tienken, Susanne: Alltagsgattungen und der Ort von Kultur. Sprachwissenschaftliche und kulturanalytische Studien anhand von Milchverpackungen in Deutschland und Schweden. Stockholm 2008.

Tienken, Susanne: »Muster - kulturanalytisch betrachtet«. In: Christa Dürscheid/Jan Georg Schneider (Hg.): Handbuch Satz, Äußerung, Schema. Berlin/Boston 2015, S. 464-484.

van der Wal, Marijke/Rutten, Gijsbert: »Ego-documents in a historical-sociolinguistic perspective«. In: Dies. (Hg.): Touching the Past: Studies in the historical sociolinguistics of ego-documents. Amsterdam 2013, S. 1-17

Wells, Christopher J.: »Schafft Sprache kulturelle Identität?« In: Andreas Gardt/ Ulrike Haß-Zumkehr/ Thorsten Roelcke (Hg): Sprachgeschichte als Kulturgeschichte. Berlin/New York 1999, S. 411-418.

Wengeler, Martin: »Linguistik als Kulturwissenschaft. Eine Einführung in diesen Band«. In: Ders. (Hg.): Linguistik als Kulturwissenschaft. Hildesheim/New York 2006, S. 1-23. 